\title{
A research on the recursive value and general terms of the analogue Euler zeta function on positive integers
}

JY Kang ${ }^{*}$ and CS Ryoo

\section{${ }^{*}$ Correspondence:}

rkdwjddnr2002@yahoo.co.kr

Department of Mathematics,

Hannam University, Daejeon,

306-791, Korea

\begin{abstract}
Our aim is to find the general term of the analogue Euler zeta function in positive integers by using Fourier series. We also figure out the generalized coefficients of Fourier series and investigate some interesting relation in the integers.
\end{abstract}

Keywords: Fourier series; cosine series; sine series; analogue Euler zeta function

\section{Introduction}

Many mathematicians have studied various kinds of an analogue zeta function such as Dirichlet $L$-function [1-28]. Friedman and Cohen constructed the $p$-adic analogue for Hurwitz zeta functions [14]. By using multiple Volkenborn integrals, Tangedal and Young defined a $p$-adic multiple zeta function and a log gamma function [1-18]. Ryoo, Kim and Kim have defined various analogue zeta functions to combine the Euler numbers and Bernoulli numbers [1-12, 17, 18].

Therefore one of the most important and fascinating functions is the zeta function in mathematics $[9,10]$. Bernhard Riemann (1826-1866) found something amazing; namely the Riemann zeta function. He recognized the importance of the function onto the entire complex plane $\mathbb{C}$ except $s=1$.

If $\operatorname{Re} z \geq 1+\epsilon$, where $\epsilon>0$, then

$$
\sum_{k=m}^{n}\left|k^{-z}\right| \leq \sum_{k=m}^{n} k^{-1-\epsilon}
$$

implies that $\sum_{n=1}^{\infty}\left|n^{-z}\right|$ converges uniformly on $\{z \in \mathbb{C} \mid \operatorname{Re} z \geq 1+\epsilon\}$.

The Riemann zeta function $\zeta_{R}(s)$ is defined usually by

$$
\zeta_{R}(s):= \begin{cases}\sum_{n=1}^{\infty} \frac{1}{n^{s}}=\frac{1}{1-2^{-s}} \sum_{n=1}^{\infty} \frac{1}{(2 n-1)^{s}} & (\operatorname{Re}(s)>1), \\ \frac{1}{1-2^{1-s}} \sum_{n=1}^{\infty} \frac{(-1)^{n-1}}{n^{s}} & (\operatorname{Re}(s)>0, s \neq 1) .\end{cases}
$$

Some values can be calculated explicitly but $\zeta_{R}(2 k+1)$, where $k=1,2, \ldots$ are still mysterious. The number $\zeta_{R}(3)$ was demonstrated to be an irrational number by Apery (French mathematician) and can be seen in Hardy, Grosswald, Zhang, Srivastava, and others [2028]. This Riemann zeta function is the Dirichlet zeta-function, the special case that arises when we take $\chi(n)=1$.

(0) 2013 Kang and Ryoo; licensee Springer. This is an Open Access article distributed under the terms of the Creative Commons Attribution License (http://creativecommons.org/licenses/by/2.0), which permits unrestricted use, distribution, and reproduction in any medium, provided the original work is properly cited. 
In 1837, Lejeune Dirichlet modified the zeta function and he separated the primes into separate categories. The primes depend on the remainder when divided by $k$. His modified zeta function is of the form

$$
L(s, \chi)=\frac{\chi(1)}{1^{s}}+\frac{\chi(2)}{2^{s}}+\frac{\chi(3)}{3^{s}}+\cdots,
$$

where $\chi(n)$ is a special kind of the function [20]. Any function of the form $L(s, \chi)$ is known as a Dirichlet $L$-series where $s$ is a real number greater than 1 and $\chi$ is called as Dirichlet character.

Mathematicians have studied extensively the Riemann zeta function and the Dirichlet zeta function because these functions play an important role in physics, complex analysis and number theory etc. They also recognized that the discovery of these zeta functions dates back to Euler.

Leonhard Euler (1707-1783) defined the zeta function for any real number greater than 1 by the infinite sum. After Euler defined this function, he showed that it had a deep and profound connection with the pattern of the primes. He also calculated $\zeta(2)=\frac{\pi^{2}}{6}$, and $\zeta(2)$ has been researched, proved by mathematicians [26]. The values of the Riemann zeta function were computed by the Euler zeta function at even positive integers. The analogue Euler zeta function replaces the Euler zeta function by mathematician's research. The Euler zeta function was originally constructed by Kim (see [3]) and Kim gave the values of the Euler zeta function as positive integers (see [3, Theorem 3.1]). Kim, Choi, and Kim researched to combine the Euler numbers and Bernoulli numbers in order to get values or a generalized term [1].

The Euler zeta function is defined as follows.

Definition 1.1 For $s=1, s \in \mathbb{C}$ and $\operatorname{Re}(s)>0$,

$$
\zeta_{E}(s)=2 \sum_{n=1}^{\infty} \frac{(-1)^{n-1}}{n^{s}} \quad(\text { see }[1-3,8])
$$

From Definition 1.1, we define the analogue Euler zeta function as follows.

Definition 1.2 Let $s=1, s \in \mathbb{C}$ and $\operatorname{Re}(s)>0$.

$$
\zeta_{A E}(s)=\sum_{n=1}^{\infty} \frac{(-1)^{n}}{n^{s}} .
$$

We easily note that $\zeta_{E}(s)=-2 \zeta_{A E}(s)$.

In this paper, we find out the generalized coefficients of Fourier series and investigate some interesting relations in the positive integers. We also investigate values and a generalized term of the analogue Euler zeta function $\zeta_{A E}(s)$ in the same way by using the Fourier series.

The Fourier series can be expressed as summation between sine series and cosine series instead of complicated functions. 
If the function $f(x)$ has period $2 p$, then the Fourier series of $f(x)$ is

$$
f(x)=\frac{a_{0}}{2}+\sum_{n=1}^{\infty}\left(a_{n} \cos \frac{n \pi x}{p}+b_{n} \sin \frac{n \pi x}{p}\right),
$$

where coefficients of the Fourier series $a_{0}, a_{n}$, and $b_{n}$ are defined by the integrals

$$
\begin{aligned}
& a_{0}=\frac{1}{p} \int_{-p}^{p} f(x) d x, \\
& a_{n}=\frac{1}{p} \int_{-p}^{p} f(x) \cos \frac{n \pi x}{p} d x, \\
& b_{n}=\frac{1}{p} \int_{-p}^{p} f(x) \sin \frac{n \pi x}{p} d x \quad \text { (see [19]). }
\end{aligned}
$$

A special instance of the Fourier series is the cosine series. If $f(x)=f(-x)$ is initially defined over the interval $[0, p]$, then it can be extended to $[-p, p]$ and then extended periodically with period $2 p$. So, the cosine series of the Fourier series on $[-p, p]$ is defined by

$$
f(x)=\frac{a_{0}}{2}+\sum_{n=1}^{\infty} a_{n} \cos \frac{n \pi x}{p}
$$

where

$$
\begin{aligned}
& a_{0}=\frac{1}{p} \int_{-p}^{p} f(x) d x \\
& a_{n}=\frac{1}{p} \int_{-p}^{p} f(x) \cos \frac{n \pi x}{p} d x, \quad \text { and in this case } \\
& b_{n}=\frac{1}{p} \int_{-p}^{p} f(x) \sin \frac{n \pi x}{p} d x=0 \quad(\text { see }[1,19]) .
\end{aligned}
$$

The sine series is a special instance of the Fourier series. Let $f(-x)=-f(x)$. Then $f(x)$ can be extended to $[-p, p]$. The Fourier series for this odd, periodic function reduces to the sine series in the form

$$
\begin{aligned}
& f(x)=\sum_{n=1}^{\infty} b_{n} \sin \frac{n \pi x}{p}, \text { with } \\
& b_{n}=\frac{1}{p} \int_{-p}^{p} f(x) \sin \frac{n \pi x}{p} d x,
\end{aligned}
$$

because $a_{0}=\frac{1}{p} \int_{-p}^{p} f(x) d x=0$, each $a_{n}=\frac{1}{p} \int_{-p}^{p} f(x) \cos \frac{n \pi x}{p} d x=0$.

We denote that $a_{0}=a_{0}^{(l)}, a_{n}=a_{n}^{(l)}$, and $b_{n}=b_{n}^{(l)}$ when $f(x)=x^{l}$.

The paper is organized as follows. In Section 2, we construct generalized coefficients of sine series and cosine series in the positive integers and prove them. We also study some interesting relations about sine series and cosine series in the positive integers. In Section 3, applying these ideas, generalized coefficients will be used to obtain the main results of this paper. We also find the general term of the analogue Euler zeta function. 


\section{The coefficient's rule of cosine series and sine series}

In this section, we construct the coefficient's rule of cosine series and sine series. We access some relations about the coefficient sine series and cosine series.

The cosine series for $f(x)$ is given by the following theorem.

Theorem 2.1 Let $f(x)=x^{l}$ for $-2<x<2$ and l be a positive even integer.

$$
\begin{aligned}
f(x) & =\frac{a_{0}}{2}+\sum_{n=1}^{\infty} a_{n} \cos \frac{n \pi x}{2}, \\
a_{0}^{(l)} & =\int_{0}^{2} x^{l} d x=\frac{2^{l+1}}{l+1}, \\
a_{n}^{(l)} & =\int_{0}^{2} x^{l} \cos \frac{n \pi x}{2} d x=2^{l+1} \cos n \pi \sum_{k=0}^{\frac{l-2}{2}}(-1)^{k} \frac{{ }_{l} P_{2 k+1}}{(n \pi)^{2(k+1)}} \\
& =2^{l+1} l ! \cos n \pi \sum_{k=0}^{\frac{l-2}{2}} \frac{(-1)^{k}}{(l-(2 k+1)) !(n \pi)^{2(k+1)}},
\end{aligned}
$$

where ${ }_{n} P_{r}=\frac{n !}{(n-r) !}$.

Proof We shall prove Theorem 2.1 using mathematical induction. We assume that $f(x)=x^{l}$ for $-2<x<2$ and $l$ is a positive even integer.

Clearly, $a_{0}^{(2)}$ and $a_{n}^{(2)}$ hold when $l=2$ as follows.

$$
\begin{aligned}
a_{0}^{(2)} & =\int_{0}^{2} x^{2} d x=\frac{2^{3}}{3}, \\
a_{n}^{(2)} & =\int_{0}^{2} x^{2} \cos \frac{n \pi x}{2} d x=\left[\frac{2}{n \pi} x^{2} \sin \frac{n \pi x}{2}\right]_{0}^{2}-\frac{2^{2}}{n \pi} \int_{0}^{2} x \sin \frac{n \pi x}{2} d x \\
& =\frac{2 \cdot 2^{3}}{n^{2} \pi^{2}} \cos n \pi .
\end{aligned}
$$

Suppose that $l=m$ is true for $a_{0}^{(m)}$ and $a_{n}^{(m)}$ in the positive even integers. That is,

$$
\begin{aligned}
a_{0}^{(m)} & =\int_{0}^{2} x^{m} d x=\frac{2^{m+1}}{m+1}, \\
a_{n}^{(m)} & =\int_{0}^{2} x^{m} \cos \frac{n \pi x}{2} d x=\left[\frac{2}{n \pi} x^{m} \sin \frac{n \pi x}{2}\right]_{0}^{2}-\frac{2 m}{n \pi} \int_{0}^{2} x^{m-1} \sin \frac{n \pi x}{2} d x \\
& =2^{m+1} \cos n \pi \sum_{k=0}^{\frac{m-2}{2}}(-1)^{k} \frac{m P_{2 k+1}}{(n \pi)^{2(k+1)}} \\
& =2^{m+1} m ! \cos n \pi \sum_{k=0}^{\frac{m-2}{2}} \frac{(-1)^{k}}{(m-(2 k+1)) !(n \pi)^{2(k+1)}} .
\end{aligned}
$$

Consider the case $l=m+2$ ( $m$ : even).

$$
a_{0}^{(m+2)}=\int_{0}^{2} x^{m+2} d x=\frac{2^{m+3}}{m+3} \quad \text { is trivial. }
$$


By the above assumption, we get

$$
\begin{aligned}
a_{n}^{(m+2)} & =\int_{0}^{2} x^{m+2} \cos \frac{n \pi x}{2} d x=\left[\frac{2}{n \pi} x^{m+2} \sin \frac{n \pi x}{2}\right]_{0}^{2}-\frac{2(m+2)}{n \pi} \int_{0}^{2} x^{m+1} \sin \frac{n \pi x}{2} d x \\
& =-\frac{2(m+2)}{n \pi} \int_{0}^{2} x^{m+1} d\left(-\frac{2}{n \pi} \cos \left(\frac{n \pi x}{2}\right)\right) \\
& =\frac{2^{m+3}(m+2)}{n^{2} \pi^{2}} \cos (n \pi)-\frac{2^{2}(m+1)(m+2)}{n^{2} \pi^{2}} \int_{0}^{2} x^{m} \cos \left(\frac{n \pi x}{2}\right) d x \\
& =\frac{2^{m+3}(m+2)}{n^{2} \pi^{2}} \cos n \pi-\frac{2^{2}(m+1)(m+2)}{n^{2} \pi^{2}}\left(2^{m+1} \cos n \pi \sum_{k=0}^{\frac{m-2}{2}}(-1)^{k} \frac{m P_{2 k+1}}{(n \pi)^{2(k+1)}}\right) \\
& =2^{m+3} \cos n \pi \sum_{k=0}^{\frac{m}{2}}(-1)^{k} \frac{m+2 P_{2 k+1}}{(n \pi)^{2(k+1)}} \\
& =2^{m+3}(m+2) ! \cos n \pi \sum_{k=0}^{\frac{m}{2}} \frac{(-1)^{k}}{(m+2-(2 k+1)) !(n \pi)^{2(k+1)}} .
\end{aligned}
$$

That is, $a_{0}^{(l)}$ and $a_{n}^{(l)}$ hold for $l=m+2(m$ : even) if it holds for $l=m$. Thus, we complete the proof of the theorem.

Remarks Let $f(x)=x^{2}$ for $-2<x<2$. Then we have the following equation:

$$
\begin{aligned}
& f(x)=\frac{a_{0}}{2}+\sum_{n=1}^{\infty} a_{n} \cos \frac{n \pi x}{2} \\
& a_{0}^{(2)}=\int_{0}^{2} x^{2} d x=\frac{2^{3}}{3} \\
& a_{n}^{(2)}=\int_{0}^{2} x^{2} \cos \frac{n \pi x}{2} d x=2^{2+1} \cos n \pi \sum_{k=0}^{\frac{2-2}{2}}(-1)^{k} \frac{{ }_{2} P_{2 k+1}}{(n \pi)^{2(k+1)}}=\frac{2 \cdot 2^{3}}{n^{2} \pi^{2}} \cos n \pi .
\end{aligned}
$$

Let $f(x)=x^{4}$ for $-2<x<2$. From Theorem 2.1, we get

$$
\begin{aligned}
f(x) & =\frac{a_{0}}{2}+\sum_{n=1}^{\infty} a_{n} \cos \frac{n \pi x}{2} \\
a_{0}^{(4)} & =\int_{0}^{2} x^{4} d x=\frac{2^{5}}{5}, \\
a_{n}^{(4)} & =\int_{0}^{2} x^{4} \cos \frac{n \pi x}{2} d x=2^{4+1} \cos n \pi \sum_{k=0}^{\frac{4-2}{2}}(-1)^{k} \frac{{ }_{4} P_{2 k+1}}{(n \pi)^{2(k+1)}} \\
& =\frac{4 \cdot 2^{5}}{n^{2} \pi^{2}} \cos n \pi-\frac{4 \cdot 3 \cdot 2 \cdot 2^{5}}{n^{4} \pi^{4}} \cos n \pi,
\end{aligned}
$$

where ${ }_{n} P_{r}=\frac{n !}{(n-r) !}=n \cdot(n-1) \cdot(n-2) \cdots(n-r+1)$. 
From (2.2), we get an interesting rule of coefficient $a_{n}^{(4)}$.

$$
a_{n}^{(4)}=2^{5} \cos n \pi\left(\begin{array}{cc}
4 & 0 \\
0 & -4 \cdot 3 \cdot 2
\end{array}\right)\left(\begin{array}{c}
\frac{1}{n^{2} \pi^{2}} \\
\frac{1}{n^{4} \pi^{4}}
\end{array}\right)=2^{5} \cos n \pi\left(\begin{array}{cc}
{ }_{4} P_{1} & 0 \\
0 & -{ }_{4} P_{3}
\end{array}\right)\left(\begin{array}{c}
\frac{1}{n^{2} \pi^{2}} \\
\frac{1}{n^{4} \pi^{4}}
\end{array}\right) .
$$

Let $f(x)=x^{6}$ for $-2<x<2$. The cosine series of $f(x)$ is the following equation.

$$
\begin{aligned}
f(x) & =\frac{a_{0}}{2}+\sum_{n=1}^{\infty} a_{n} \cos \frac{n \pi x}{2}, \\
a_{0}^{(6)} & =\int_{0}^{2} x^{6} d x=\frac{2^{7}}{7} \\
a_{n}^{(6)} & =\int_{0}^{2} x^{6} \cos \frac{n \pi x}{2} d x=2^{6+1} \cos n \pi \sum_{k=0}^{\frac{6-2}{2}}(-1)^{k} \frac{{ }_{6} P_{2 k+1}}{(n \pi)^{2(k+1)}} \\
& =\frac{6 \cdot 2^{7}}{n^{2} \pi^{2}} \cos n \pi-\frac{6 \cdot 5 \cdot 4 \cdot 2^{7}}{n^{4} \pi^{4}} \cos n \pi+\frac{6 \cdot 5 \cdot 4 \cdot 3 \cdot 2 \cdot 2^{7}}{n^{6} \pi^{6}} \cos n \pi,
\end{aligned}
$$

where ${ }_{n} P_{r}=\frac{n !}{(n-r) !}=n \cdot(n-1) \cdot(n-2) \cdots(n-r+1)$.

From (2.3), we are able to express the rule of coefficient $a_{n}^{(6)}$ by using the matrix

$$
\begin{aligned}
a_{n}^{(6)} & =2^{7} \cos n \pi\left(\begin{array}{ccc}
6 & 0 & 0 \\
0 & -6 \cdot 5 \cdot 4 & 0 \\
0 & 0 & 6 \cdot 5 \cdot 4 \cdot 3 \cdot 2
\end{array}\right)\left(\begin{array}{c}
\frac{1}{n^{2} \pi^{2}} \\
\frac{1}{n^{4} \pi^{4}} \\
\frac{1}{n^{6} \pi^{6}}
\end{array}\right) \\
& =2^{7} \cos n \pi\left(\begin{array}{ccc}
{ }^{6} P_{1} & 0 & 0 \\
0 & -{ }_{6} P_{3} & 0 \\
0 & 0 & { }_{6} P_{5}
\end{array}\right)\left(\begin{array}{c}
\frac{1}{n^{2} \pi^{2}} \\
\frac{1}{n^{4} \pi^{4}} \\
\frac{1}{n^{6} \pi^{6}}
\end{array}\right) .
\end{aligned}
$$

Let $f(x)=x^{8}$ for $-2<x<2$. Then the cosine series of $f(x)$ is the following equation:

$$
\begin{aligned}
f(x)= & \frac{a_{0}}{2}+\sum_{n=1}^{\infty} a_{n} \cos \frac{n \pi x}{2}, \\
a_{0}^{(8)}= & \int_{0}^{2} x^{8} d x=\frac{2^{9}}{9}, \\
a_{n}^{(8)}= & \int_{0}^{2} x^{8} \cos \frac{n \pi x}{2} d x=2^{8+1} \cos n \pi \sum_{k=0}^{\frac{8-2}{2}}(-1)^{k} \frac{{ }_{8} P_{2 k+1}}{(n \pi)^{2(k+1)}} \\
= & \frac{8 \cdot 2^{9}}{n^{2} \pi^{2}} \cos n \pi-\frac{8 \cdot 7 \cdot 6 \cdot 2^{9}}{n^{4} \pi^{4}} \cos n \pi+\frac{8 \cdot 7 \cdot 6 \cdot 5 \cdot 4 \cdot 2^{9}}{n^{6} \pi^{6}} \cos n \pi \\
& -\frac{8 \cdot 7 \cdot 6 \cdot 5 \cdot 4 \cdot 3 \cdot 2 \cdot 2^{9}}{n^{8} \pi^{8}} \cos n \pi,
\end{aligned}
$$

where ${ }_{n} P_{r}=\frac{n !}{(n-r) !}=n \cdot(n-1) \cdot(n-2) \cdots(n-r+1)$. 
From the above equation, we are able to represent the rule of coefficient $a_{n}^{(8)}$ by using the matrix

$$
\begin{aligned}
a_{n}^{(8)} & =2^{9} \cos n \pi\left(\begin{array}{cccc}
8 & 0 & 0 & 0 \\
0 & -8 \cdot 7 \cdot 6 & 0 & 0 \\
0 & 0 & 8 \cdot 7 \cdot 6 \cdot 5 \cdot 4 & 0 \\
0 & 0 & 0 & -8 \cdot 7 \cdot 6 \cdot 5 \cdot 4 \cdot 3 \cdot 2
\end{array}\right)\left(\begin{array}{c}
\frac{1}{n^{2} \pi^{2}} \\
\frac{1}{n^{4} \pi^{4}} \\
\frac{1}{n^{6} \pi^{6}} \\
\frac{1}{n^{8} \pi^{8}}
\end{array}\right) \\
& =2^{9} \cos n \pi\left(\begin{array}{cccc}
{ }^{8} P_{1} & 0 & 0 & 0 \\
0 & -{ }_{8} P_{3} & 0 & 0 \\
0 & 0 & { }_{8} P_{5} & 0 \\
0 & 0 & 0 & -{ }_{8} P_{7}
\end{array}\right)\left(\begin{array}{l}
\frac{1}{n^{2} \pi^{2}} \\
\frac{1}{n^{4} \pi^{4}} \\
\frac{1}{n^{6} \pi^{6}} \\
\frac{1}{n^{8} \pi^{8}}
\end{array}\right) .
\end{aligned}
$$

By using the matrix, we can display the coefficient $a_{n}^{(l)}$ of cosine series in Theorem 2.1 as follows.

Corollary 2.2 Let l be a positive even integer. Then one has

$$
a_{n}^{(l)}=2^{l+1} \cos n \pi\left(\begin{array}{ccccc}
{ }_{l} P_{1} & 0 & 0 & 0 & \ldots \\
0 & -{ }_{l} P_{3} & 0 & 0 & \ldots \\
0 & 0 & { }_{l} P_{5} & 0 & \ldots \\
0 & 0 & 0 & -{ }_{l} P_{7} & \ldots \\
\vdots & \vdots & \vdots & \vdots & \ddots
\end{array}\right)\left(\begin{array}{c}
\frac{1}{n^{2} \pi^{2}} \\
\frac{1}{n^{4} \pi^{4}} \\
\frac{1}{n^{6} \pi^{6}} \\
\frac{1}{n^{8} \pi^{8}} \\
\vdots
\end{array}\right) .
$$

From now on, we will see the coefficients of the sine series. The sine series $f(x)$ is given by the following theorem.

Theorem 2.3 Let $f(x)=x^{l}$ for $-2<x<2$ and $l$ be a positive odd integer.

$$
\begin{aligned}
f(x) & =\sum_{n=1}^{\infty} b_{n} \sin \frac{n \pi x}{2}, \\
b_{n}^{(l)} & =\int_{0}^{2} x^{l} \sin \frac{n \pi x}{2} d x=2^{l+1} \cos n \pi \sum_{k=0}^{\frac{l-1}{2}}(-1)^{k+1} \frac{{ }_{l} P_{2 k}}{(n \pi)^{2 k+1}} \\
& =2^{l+1} l ! \cos n \pi \sum_{k=0}^{\frac{l-1}{2}} \frac{(-1)^{k+1}}{(l-2 k) !(n \pi)^{2 k+1}},
\end{aligned}
$$

when ${ }_{n} P_{r}=\frac{n !}{(n-r) !}$.

Proof (By mathematical induction) Where $l=1, b_{n}^{(l)}$ says that

$$
\begin{aligned}
b_{n}^{(1)} & =\int_{0}^{2} x^{1} \sin \frac{n \pi x}{2} d x=\left[-\frac{2}{n \pi} x \cos \frac{n \pi x}{2}\right]_{0}^{2}+\frac{2}{n \pi} \int_{0}^{2} \cos \frac{n \pi x}{2} d x \\
& =-\frac{2^{2}}{n \pi} \cos n \pi+\frac{2}{n \pi}\left[\frac{2}{n \pi} \sin \frac{n \pi x}{2}\right]_{0}^{2}=-\frac{2^{2}}{n \pi} \cos n \pi,
\end{aligned}
$$

which is true. 
Now fix that $l=m(m$ : odd $)$ is true for $b_{n}^{(m)}$ and suppose that $b_{n}^{(m)}$ holds, that is,

$$
\begin{aligned}
b_{n}^{(m)} & =\int_{0}^{2} x^{m} \sin \frac{n \pi x}{2} d x=-\frac{2^{m+1}}{n \pi} \cos n \pi+\frac{2 m}{n \pi} \int_{0}^{2} x^{m-1} \cos \frac{n \pi x}{2} d x \\
& =2^{m+1} \cos n \pi \sum_{k=0}^{\frac{m-1}{2}}(-1)^{k+1} \frac{m P_{2 k}}{(n \pi)^{2 k+1}} \\
& =2^{m+1} m ! \cos n \pi \sum_{k=0}^{\frac{m-1}{2}} \frac{(-1)^{k+1}}{(m-2 k) !(n \pi)^{2 k+1}} .
\end{aligned}
$$

By our hypothesis on $b_{n}^{(m)}$, we see that

$$
\begin{aligned}
b_{n}^{(m+2)} & =\int_{0}^{2} x^{m+2} \sin \frac{n \pi x}{2} d x \\
& =-\frac{2^{m+3}}{n \pi} \cos n \pi+\frac{2(m+2)}{n \pi} \int_{0}^{2} x^{m+1} \cos \frac{n \pi x}{2} d x \\
& =-\frac{2^{m+3}}{n \pi} \cos n \pi-\frac{2^{2}(m+1)(m+2)}{n \pi} \int_{0}^{2} x^{m} \sin \frac{n \pi x}{2} d x \\
& =-\frac{2^{m+3}}{n \pi} \cos n \pi-\frac{2^{2}(m+1)(m+2)}{n \pi}\left(2^{m+1} \cos n \pi \sum_{k=0}^{\frac{m-1}{2}}(-1)^{k+1} \frac{m P_{2 k}}{(n \pi)^{2 k+1}}\right) \\
& =2^{m+3} \cos n \pi \sum_{k=0}^{\frac{m+1}{2}}(-1)^{k+1} \frac{m+2}{(n \pi)^{2 k+1}} \\
& =2^{m+3}(m+2) ! \cos n \pi \sum_{k=0}^{\frac{m+1}{2}} \frac{(-1)^{k+1}}{(m+2-2 k) !(n \pi)^{2 k+1}} .
\end{aligned}
$$

Therefore $b_{n}$ holds for $l=m+2$ ( $m$ : odd).

Thus, we conclude the proof of the theorem by the principle of mathematical induction.

Let $f(x)=x$ for $-2<x<2$. Then we have the following equation:

$$
\begin{aligned}
f(x) & =\sum_{n=1}^{\infty} b_{n} \sin \frac{n \pi x}{2} \\
b_{n}^{(1)} & =\int_{0}^{2} x^{1} \sin \frac{n \pi x}{2} d x=2^{1+1} \cos n \pi \sum_{k=0}^{\frac{1-1}{2}}(-1)^{k+1} \frac{{ }_{1} P_{2 k}}{(n \pi)^{2 k+1}} \\
& =-\frac{2^{2}}{n \pi} \cos n \pi .
\end{aligned}
$$

Let $f(x)=x^{3}$ for $-2<x<2$. From Theorem 2.3, we get

$$
f(x)=\sum_{n=1}^{\infty} b_{n} \sin \frac{n \pi x}{2}
$$




$$
\begin{aligned}
b_{n}^{(3)} & =\int_{0}^{2} x^{3} \sin \frac{n \pi x}{2} d x=2^{3+1} \cos n \pi \sum_{k=0}^{\frac{3-1}{2}}(-1)^{k+1} \frac{{ }_{3} P_{2 k}}{(n \pi)^{2 k+1}} \\
& =-\frac{2^{4}}{n \pi} \cos n \pi+\frac{3 \cdot 2 \cdot 2^{4}}{n^{3} \pi^{3}} \cos n \pi,
\end{aligned}
$$

where ${ }_{n} P_{r}=\frac{n !}{(n-r) !}=n \cdot(n-1) \cdot(n-2) \cdots(n-r+1)$.

From (2.5), we also get an interesting rule of coefficient $b_{n}$.

$$
b_{n}^{(3)}=2^{4} \cos n \pi\left(\begin{array}{cc}
-1 & 0 \\
0 & 3 \cdot 2
\end{array}\right)\left(\begin{array}{c}
\frac{1}{n \pi} \\
\frac{1}{n^{3} \pi^{3}}
\end{array}\right)=2^{4} \cos n \pi\left(\begin{array}{cc}
3 P_{0} & 0 \\
0 & { }_{3} P_{2}
\end{array}\right)\left(\begin{array}{c}
\frac{1}{n \pi} \\
\frac{1}{n^{3} \pi^{3}}
\end{array}\right) .
$$

Let $f(x)=x^{5}$ for $-2<x<2$. The sine series of $f(x)$ is the following equation:

$$
\begin{aligned}
f(x) & =\sum_{n=1}^{\infty} b_{n} \sin \frac{n \pi x}{2}, \\
b_{n}^{(5)} & =\int_{0}^{2} x^{5} \sin \frac{n \pi x}{2} d x=2^{5+1} \cos n \pi \sum_{k=0}^{\frac{5-1}{2}}(-1)^{k+1} \frac{{ }_{5} P_{2 k}}{(n \pi)^{2 k+1}} \\
& =-\frac{2^{6}}{n \pi} \cos n \pi+\frac{5 \cdot 4 \cdot 2^{6}}{n^{3} \pi^{3}} \cos n \pi-\frac{5 \cdot 4 \cdot 3 \cdot 2 \cdot 2^{6}}{n^{3} \pi^{3}} \cos n \pi,
\end{aligned}
$$

where ${ }_{n} P_{r}=\frac{n !}{(n-r) !}=n \cdot(n-1) \cdot(n-2) \cdots(n-r+1)$.

From (2.6), we are able to express the rule of coefficient $b_{n}^{(5)}$ by using the matrix

$$
\begin{aligned}
b_{n}^{(5)} & =2^{6} \cos n \pi\left(\begin{array}{ccc}
-1 & 0 & 0 \\
0 & 5 \cdot 4 & 0 \\
0 & 0 & -5 \cdot 4 \cdot 3 \cdot 2
\end{array}\right)\left(\begin{array}{c}
\frac{1}{n \pi} \\
\frac{1}{n^{3} \pi^{3}} \\
\frac{1}{n^{5} \pi^{5}}
\end{array}\right) \\
& =2^{6} \cos n \pi\left(\begin{array}{ccc}
-5 P_{0} & 0 & 0 \\
0 & { }_{5} P_{2} & 0 \\
0 & 0 & -{ }_{5} P_{6}
\end{array}\right)\left(\begin{array}{c}
\frac{1}{n \pi} \\
\frac{1}{n^{3} \pi^{3}} \\
\frac{1}{n^{5} \pi^{5}}
\end{array}\right) .
\end{aligned}
$$

Let $f(x)=x^{7}$ for $-2<x<2$. Then the sine series of $f(x)$ is the following equation:

$$
\begin{aligned}
f(x)= & \sum_{n=1}^{\infty} b_{n} \sin \frac{n \pi x}{2}, \\
b_{n}^{(7)}= & \int_{0}^{2} x^{7} \sin \frac{n \pi x}{2} d x=2^{7+1} \cos n \pi \sum_{k=0}^{\frac{7-1}{2}}(-1)^{k+1} \frac{7 P_{2 k}}{(n \pi)^{2 k+1}} \\
= & -\frac{2^{8}}{n \pi} \cos n \pi+\frac{7 \cdot 6 \cdot 2^{8}}{n^{3} \pi^{3}} \cos n \pi-\frac{7 \cdot 6 \cdot 5 \cdot 4 \cdot 2^{8}}{n^{5} \pi^{5}} \cos n \pi \\
& +\frac{7 \cdot 6 \cdot 5 \cdot 4 \cdot 3 \cdot 2 \cdot 2^{8}}{n^{7} \pi^{7}} \cos n \pi,
\end{aligned}
$$

where ${ }_{n} P_{r}=\frac{n !}{(n-r) !}=n \cdot(n-1) \cdot(n-2) \cdots(n-r+1)$. 
From the above equation, we are able to represent the rule of coefficient $b_{n}$ by using the matrix

$$
\begin{aligned}
b_{n}^{(7)} & =2^{8} \cos n \pi\left(\begin{array}{cccc}
-1 & 0 & 0 & 0 \\
0 & 7 \cdot 6 & 0 & 0 \\
0 & 0 & -7 \cdot 6 \cdot 5 \cdot 4 & 0 \\
0 & 0 & 0 & 7 \cdot 6 \cdot 5 \cdot 4 \cdot 3 \cdot 2
\end{array}\right)\left(\begin{array}{c}
\frac{1}{n \pi} \\
\frac{1}{n^{3} \pi^{3}} \\
\frac{1}{n^{5} \pi^{5}} \\
\frac{1}{n^{7} \pi^{7}}
\end{array}\right) \\
& =2^{8} \cos n \pi\left(\begin{array}{cccc}
-{ }_{7} P_{0} & 0 & 0 & 0 \\
0 & { }_{7} P_{2} & 0 & 0 \\
0 & 0 & -{ }_{7} P_{4} & 0 \\
0 & 0 & 0 & { }_{7} P_{6}
\end{array}\right)\left(\begin{array}{c}
\frac{1}{n \pi} \\
\frac{1}{n^{3} \pi^{3}} \\
\frac{1}{n^{5} \pi^{5}} \\
\frac{1}{n^{7} \pi^{7}}
\end{array}\right) .
\end{aligned}
$$

By using the matrix, we can arrange the coefficient $b_{n}^{(l)}$ of sine series in Theorem 2.3 as follows.

Corollary 2.4 Let l be any positive odd integer. Then we get

$$
b_{n}^{(l)}=2^{l+1} \cos n \pi\left(\begin{array}{ccccc}
-P_{0} & 0 & 0 & 0 & \ldots \\
0 & { }_{l} P_{2} & 0 & 0 & \ldots \\
0 & 0 & -{ }_{l} P_{4} & 0 & \ldots \\
0 & 0 & 0 & { }_{l} P_{6} & \ldots \\
\vdots & \vdots & \vdots & \vdots & \ddots
\end{array}\right)\left(\begin{array}{c}
\frac{1}{n \pi} \\
\frac{1}{n^{3} \pi^{3}} \\
\frac{1}{n^{5} \pi^{5}} \\
\frac{1}{n^{7} \pi^{7}} \\
\vdots
\end{array}\right) .
$$

From Theorem 2.1 and Theorem 2.3, we get the relation of coefficients between $a_{n}^{(l)}$ and $b_{n}^{(l)}$.

Theorem 2.5 Let $a_{n}^{(l)}$ : the coefficient of cosine series, $b_{n}^{(l)}$ : the coefficient of sine series, and $m \in \mathbb{N}$.

$$
b_{n}^{(l)}=-\frac{n \pi}{4 m} a_{n}^{(l+1)}, \quad \text { where } l=2 m-1, m \in \mathbb{N} .
$$

Proof Take $l=2 m-1(m \in \mathbb{N})$ in Theorem 2.3. Then we easily see that

$$
\begin{aligned}
b_{n}^{(2 m-1)} & =2^{2 m} \cos n \pi \sum_{k=0}^{m-1} \frac{(-1)^{k+1}(2 m-1) !}{(2 m-2 k-1) !(n \pi)^{2 k+1}} \\
& =2^{2 m+1} \cos n \pi \sum_{k=0}^{m-1} \frac{(-1)^{k}(2 m) !}{(2 m-2 k-1) !(n \pi)^{2(k+1)}} \frac{(-1) n \pi}{4 m} .
\end{aligned}
$$

We also use $l=2 m(m \in \mathbb{N})$ from Theorem 2.1. Then we obtain the following equation:

$$
a_{n}^{(2 m)}=2^{2 m+1} \cos n \pi \sum_{k=0}^{m-1} \frac{(-1)^{k}(2 m) !}{(2 m-2 k-1) !(n \pi)^{2(k+1)}} .
$$

If $l$ is an even integer, then $b_{n}^{(l)}=0$. 
Hence,

$$
b_{n}^{(l)}=-\frac{n \pi}{4 m} a_{n}^{(l+1)}, \quad \text { where } l=2 m-1, m \in \mathbb{N} .
$$

Example 2.6 We state the relation of $l=3$ and $l=4$. Let $a_{n}^{(4)}$ : the coefficient of cosine series, $b_{n}^{(3)}$ : the coefficient of sine series, and $m \in \mathbb{N}$. By using Theorem 2.5 , we derive that

$$
\begin{aligned}
b_{n}^{(3)} & =2^{4} \cos n \pi\left(\frac{{ }_{3} P_{2}}{n^{3} \pi^{3}}-\frac{1}{n \pi}\right)=-\frac{n \pi}{8} 2^{5} \cos n \pi\left(\frac{{ }_{4} P_{3}}{n^{4} \pi^{4}}-\frac{{ }_{4} P_{1}}{n^{2} \pi^{2}}\right) \\
& =-\frac{n \pi}{4 m} a_{n}^{(4)} \quad \text { for } m=2 .
\end{aligned}
$$

\section{The analogue Euler zeta function in the integers}

In this section, we get the value and generalized term of the analogue Euler zeta function. We derive $\zeta_{A E}(2), \zeta_{A E}(4), \ldots, \zeta_{A E}(m)$ by using the coefficient of cosine series and obtain $\sum_{n=1}^{\infty} \frac{(-1)^{n}}{(2 n-1)^{m}}$ using the coefficient of sine series.

Using $f(x)=x^{l}$, Theorem 2.1 can be written as

$$
x^{l}=\frac{2^{l}}{l+1}+\sum_{n=1}^{\infty} 2^{l+1} \sum_{k=0}^{\frac{l-2}{2}}(-1)^{k} \frac{{ }_{l} P_{2 k+1}}{(n \pi)^{2(k+1)}} \cos n \pi \cos \frac{n \pi x}{2} .
$$

Taking $x=1$ in (3.1), we easily find the following equation:

$$
1=\frac{2^{l}}{l+1}+2^{l+1} \sum_{k=0}^{\frac{l-2}{2}}(-1)^{k} \frac{l^{P_{2 k+1}}}{\pi^{2(k+1)}} \sum_{n=1}^{\infty} \frac{(-1)^{n}}{(2 n)^{2(k+1)}}
$$

since $\cos (n \pi) \cos \left(\frac{n \pi}{2}\right)$ is $(-1)^{m}$ if $n=2 m$ and 0 if $n=2 m-1$ with $m \in \mathbb{N}$. Therefore, we have the results as follows:

$$
\begin{aligned}
\frac{1}{2^{l+1}}-\frac{1}{2(l+1)} & =\sum_{k=0}^{\frac{l-2}{2}}(-1)^{k} \frac{{ }_{l} P_{2 k+1}}{\pi^{2(k+1)}} \sum_{n=1}^{\infty} \frac{(-1)^{n}}{(2 n)^{2(k+1)}} \\
& =l ! \sum_{k=0}^{\frac{l-2}{2}} \frac{(-1)^{k}}{(l-2 k-1) !(2 \pi)^{2(k+1)}} \sum_{n=1}^{\infty} \frac{(-1)^{n}}{n^{2(k+1)}} .
\end{aligned}
$$

From (3.3), we get some analogue Euler zeta functions as follows.

$$
\zeta_{A E}(2)=\sum_{n=1}^{\infty} \frac{(-1)^{n}}{n^{2}}=(2 \pi)^{2} \frac{1}{{ }_{2} P_{1}}\left(\frac{1}{2^{3}}-\frac{1}{2 \cdot 3}\right)=(2 \pi)^{2}\left\{Z_{(2,1)}\left(\frac{1}{2^{2}}-\frac{1}{2 \cdot 3}\right)\right\}=-\frac{1}{12} \pi^{2}
$$

where $Z_{(2,1)}=\frac{1}{{ }_{2} P_{1}}$

$$
\begin{aligned}
\zeta_{A E}(4) & =\sum_{n=1}^{\infty} \frac{(-1)^{n}}{n^{4}}=(2 \pi)^{4}\left\{\frac{{ }_{4} P_{1}}{{ }_{4} P_{3}} \frac{1}{{ }_{2} P_{1}}\left(\frac{1}{2^{3}}-\frac{1}{2 \cdot 3}\right)-\frac{1}{{ }_{4} P_{3}}\left(\frac{1}{2^{5}}-\frac{1}{2 \cdot 5}\right)\right\} \\
& =(2 \pi)^{4}\left\{Z_{(4,1)}\left(\frac{1}{2^{3}}-\frac{1}{2 \cdot 3}\right)+Z_{(4,2)}\left(\frac{1}{2^{5}}-\frac{1}{2 \cdot 5}\right)\right\}=-\frac{7}{720} \pi^{4},
\end{aligned}
$$


where

$$
\begin{aligned}
Z_{(4,1)=} & \frac{{ }_{4} P_{1}}{{ }_{4} P_{3}} \frac{1}{{ }_{2} P_{1}}=\frac{1}{{ }_{4} P_{3}}{ }_{4} P_{1} Z_{(2,1)}, \\
Z_{(4,2)}= & -\frac{1}{{ }_{4} P_{3}}, \\
\zeta_{A E}(6)= & \sum_{n=1}^{\infty} \frac{(-1)^{n}}{n^{6}}=(2 \pi)^{6}\left\{\left(-\frac{{ }_{6} P_{1}}{{ }_{6} P_{5}} \frac{1}{{ }_{2} P_{1}}+\frac{{ }_{6} P_{3}}{{ }_{6} P_{5}} \frac{{ }_{4} P_{1}}{{ }_{4} P_{3}} \frac{1}{{ }_{2} P_{1}}\right)\left(\frac{1}{2^{3}}-\frac{1}{2 \cdot 3}\right)\right. \\
& \left.\quad+\frac{{ }_{6} P_{3}}{{ }_{6} P_{5}} \frac{-1}{{ }_{4} P_{3}}\left(\frac{1}{2^{5}}-\frac{1}{2 \cdot 5}\right)+\frac{1}{{ }_{6} P_{5}}\left(\frac{1}{2^{7}}-\frac{1}{2 \cdot 7}\right)\right\} \\
= & (2 \pi)^{6}\left\{Z_{(6,1)}\left(\frac{1}{2^{3}}-\frac{1}{2 \cdot 3}\right)+Z_{(6,2)}\left(\frac{1}{2^{5}}-\frac{1}{2 \cdot 5}\right)+Z_{(6,3)}\left(\frac{1}{2^{7}}-\frac{1}{2 \cdot 7}\right)\right\},
\end{aligned}
$$

where

$$
\begin{aligned}
& Z_{(6,1)}=-\frac{{ }_{6} P_{1}}{{ }_{6} P_{5}} \frac{1}{{ }_{2} P_{1}}+\frac{{ }_{6} P_{3}}{{ }_{6} P_{5}} \frac{P_{1}}{{ }_{4} P_{3}} \frac{1}{{ }_{2} P_{1}}=\frac{1}{{ }_{6} P_{5}}\left({ }_{6} P_{1} Z_{(2,1)}+{ }_{6} P_{3} Z_{(4,1)}\right), \\
& Z_{(6,2)}=\frac{{ }_{6} P_{3}}{{ }_{6} P_{5}} \frac{-1}{{ }_{4} P_{3}}=\frac{1}{{ }_{6} P_{5}}{ }_{6} P_{3} Z_{(4,2)}, \\
& Z_{(6,3)}=\frac{1}{{ }_{6} P_{5}} .
\end{aligned}
$$

Hence, we find out the following generalized term $\zeta(m)$, when $m$ is a positive even integer.

$$
\begin{aligned}
\zeta_{A E}(m)=\sum_{n=1}^{\infty} \frac{(-1)^{n}}{n^{m}}= & (2 \pi)^{m}\left\{Z_{(m, 1)}\left(\frac{1}{2^{3}}-\frac{1}{2 \cdot 3}\right)+Z_{(m, 2)}\left(\frac{1}{2^{5}}-\frac{1}{2 \cdot 5}\right)\right. \\
& \left.+Z_{(m, 3)}\left(\frac{1}{2^{7}}-\frac{1}{2 \cdot 7}\right)+\cdots+Z_{\left(m, \frac{m}{2}\right)}\left(\frac{1}{2^{m+1}}-\frac{1}{2 \cdot(m+1)}\right)\right\},
\end{aligned}
$$

where recursively we define (for an even positive integer $m$ )

$$
\begin{aligned}
& Z_{(m, 1)}=\sum_{k=0}^{\frac{m-4}{2}} \frac{(-1)^{\frac{m-4}{2}+k}}{(m-(2 k+1)) !} Z_{(2(k+1), 1)} \\
& Z_{(m, 2)}=\sum_{k=0}^{\frac{m-6}{2}} \frac{(-1)^{\frac{m-6}{2}+k}}{(m-(2 k+3)) !} Z_{(2(k+2), 2)}, \\
& Z_{(m, 3)}=\sum_{k=0}^{\frac{m-8}{2}} \frac{(-1)^{\frac{m-8}{2}+k}}{(m-(2 k+5)) !} Z_{(2(k+3), 3)}, \\
& \vdots \\
& Z_{\left(m, \frac{m-1}{2}\right)}=\frac{1}{3 !} Z_{\left(m-2, \frac{m-1}{2}\right)} \\
& Z_{\left(m, \frac{m+1}{2}\right)}=(-1)^{\frac{m+1}{2}} \frac{1}{m !} .
\end{aligned}
$$

From the above, we obtain the following theorem. 
Theorem 3.1 Let $m$ be any positive even integer. Then we have

$$
\begin{aligned}
\sum_{n=1}^{\infty} \frac{(-1)^{n}}{n^{m}}= & (2 \pi)^{m}\left\{\sum_{j=1}^{\frac{m-2}{2}}\left(\sum_{k=0}^{\frac{m-2 j-2}{2}} \frac{(-1)^{\frac{m-2 j-2}{2}+k} Z_{(2(k+j), j)}}{(m-2(k+j)+1) !}\right)\left(\frac{1}{2^{2 j+1}}-\frac{1}{2(2 j+1)}\right)\right. \\
& \left.+Z_{\left(m, \frac{m}{2}\right)}\left(\frac{1}{2^{m+1}}-\frac{1}{2(m+1)}\right)\right\},
\end{aligned}
$$

where $Z_{\left(m, \frac{m}{2}\right)}=(-1)^{\frac{m}{2}+1} \frac{1}{m !}$.

By observing Theorem 3.1, we can easily understand the relation between the Euler zeta function and the analogue Euler zeta function.

Example 3.2 From Theorem 3.1, in case of $m=6$ we derive that

$$
\begin{aligned}
\sum_{n=1}^{\infty} \frac{(-1)^{n}}{n^{6}}= & (2 \pi)^{6}\left\{\sum_{j=1}^{\frac{6-2}{2}}\left(\sum_{k=0}^{\frac{6-2 j-2}{2}} \frac{(-1)^{\frac{6-2 j-2}{2}+k} Z_{(2(k+j), j)}}{(6-2(k+j)+1) !}\right)\left(\frac{1}{2^{2 j+1}}-\frac{1}{2(2 j+1)}\right)\right. \\
& \left.+Z_{\left(6, \frac{6}{2}\right)}\left(\frac{1}{2^{6+1}}-\frac{1}{2(6+1)}\right)\right\} \\
= & (2 \pi)^{6}\left\{\left(\sum_{k=0} \frac{(-1)^{1+k} Z_{(2 k+2,1)}}{(5-2 k) !}\right)\left(\frac{1}{2^{3}}-\frac{1}{2 \cdot 3}\right)+\frac{Z_{(4,2)}}{3 !}\left(\frac{1}{2^{5}}-\frac{1}{2 \cdot 5}\right)\right. \\
& \left.+Z_{(6,3)}\left(\frac{1}{2^{7}}-\frac{1}{2 \cdot 7}\right)\right\} \\
= & (2 \pi)^{6}\left\{\left(\frac{-Z_{(2,1)}}{5 !}+\frac{Z_{(4,1)}}{3 !}\right)\left(\frac{1}{2^{3}}-\frac{1}{2 \cdot 3}\right)+\frac{Z_{(4,2)}}{3 !}\left(\frac{1}{2^{5}}-\frac{1}{2 \cdot 5}\right)\right. \\
& \left.+Z_{(6,3)}\left(\frac{1}{2^{7}}-\frac{1}{2 \cdot 7}\right)\right\},
\end{aligned}
$$

where $Z_{(6,3)}=\frac{1}{6 !}$.

By Theorem 2.3, we note that

$$
x^{l}=2^{l+1} \sum_{n=1}^{\infty} \sum_{k=0}^{\frac{l-1}{2}}(-1)^{k+1} \frac{{ }_{l} P_{2 k}}{(n \pi)^{2 k+1}} \cos n \pi \sin \frac{n \pi x}{2} .
$$

Let $x=1$, then we have the following equation:

$$
1=2^{l+1} \sum_{k=0}^{\frac{l-1}{2}}(-1)^{k+1} \frac{{ }_{l} P_{2 k}}{\pi^{2 k+1}} \sum_{n=1}^{\infty} \frac{(-1)^{n}}{(2 n-1)^{2 k+1}},
$$

since $\cos (n \pi) \sin \left(\frac{n \pi}{2}\right)$ is $(-1)^{m}$ if $n=2 m-1$ and 0 if $n=2 m$ with $m \in \mathbb{N}$. 
Hence, we have equation (3.6) from (3.5).

$$
\begin{aligned}
\frac{1}{2^{l+1}} & =\sum_{k=0}^{\frac{l-1}{2}}(-1)^{k+1} \frac{{ }_{l} P_{2 k}}{\pi^{2 k+1}} \sum_{n=1}^{\infty} \frac{(-1)^{n}}{(2 n-1)^{2 k+1}} \\
& =l ! \sum_{k=0}^{\frac{l-1}{2}} \frac{(-1)^{k+1}}{(l-2 k) ! \pi^{2 k+1}} \sum_{n=1}^{\infty} \frac{(-1)^{n}}{(2 n-1)^{2 k+1}} .
\end{aligned}
$$

From (3.6) with $l=1$, we obtain the following.

$$
\begin{aligned}
\sum_{n=1}^{\infty} \frac{(-1)^{n}}{(2 n-1)} & =\pi\left(-\frac{1}{{ }_{1} P_{0}}\right) \frac{1}{2^{2}}=\pi Z_{(1,1)} \frac{1}{2^{2}} \\
& =\frac{(-1)}{4} \pi,
\end{aligned}
$$

where $Z_{(1,1)}=-\frac{1}{{ }_{1} P_{0}}$.

$$
\begin{aligned}
\sum_{n=1}^{\infty} \frac{(-1)^{n}}{(2 n-1)^{3}} & =\pi^{3}\left\{\left(\frac{{ }_{3} P_{0}}{{ }_{3} P_{2}} \frac{-1}{{ }_{1} P_{0}}\right) \frac{1}{2^{2}}+\frac{1}{{ }_{3} P_{2}} \frac{1}{2^{4}}\right\} \\
& =\pi^{3}\left\{Z_{(3,1)} \frac{1}{2^{2}}+Z_{(3,2)} \frac{1}{2^{4}}\right\} \\
& =-\frac{1}{32} \pi^{3},
\end{aligned}
$$

where

$$
\begin{aligned}
& Z_{(3,1)}=\frac{{ }_{3} P_{0}}{{ }_{3} P_{2}} \frac{-1}{{ }_{1} P_{0}}=\frac{1}{{ }_{3} P_{2}}{ }_{3} P_{0} Z_{(1,1)}, \\
& \sum_{n=1} \frac{1}{{ }_{3} P_{2}}, \\
& \sum_{n=1}^{\infty} \frac{(-1)^{n}}{(2 n-1)^{5}}=\pi^{5}\left\{\left(-\frac{{ }_{5} P_{0}}{{ }_{5} P_{4}} \frac{-1}{{ }_{1} P_{0}}+\frac{{ }_{5} P_{2}}{{ }_{5} P_{4} P_{3} P_{0}} \frac{-1}{{ }_{1} P_{0}}\right) \frac{1}{2^{2}}-\left(\frac{{ }_{5} P_{2}}{{ }_{5} P_{4}} \frac{1}{{ }_{3} P_{2}}\right) \frac{1}{2^{4}}-\frac{1}{{ }_{5} P_{4}} \frac{1}{2^{4}}\right\} \\
& =\pi^{5}\left\{Z_{(5,1)} \frac{1}{2^{2}}+Z_{(5,2)} \frac{1}{2^{4}}+Z_{(5,3)} \frac{1}{2^{6}}\right\} \\
& =-\frac{7}{512} \pi^{5},
\end{aligned}
$$

where

$$
\begin{aligned}
& Z_{(5,1)}=-\frac{{ }_{5} P_{0}}{{ }_{5} P_{4}} \frac{-1}{{ }_{1} P_{0}}+\frac{{ }_{5} P_{2}}{{ }_{5} P_{4} P_{0}} \frac{-1}{{ }_{3} P_{2}} \frac{-1}{{ }_{1} P_{0}}=\frac{1}{{ }_{5} P_{4}}\left(-{ }_{5} P_{0} Z_{(1,1)}+{ }_{5} P_{2} Z_{(3,1)}\right), \\
& Z_{(5,2)}=-\frac{{ }_{5} P_{2}}{{ }_{5} P_{4}} \frac{1}{{ }_{3} P_{2}}=\frac{1}{{ }_{5} P_{4}}\left(-{ }_{5} P_{2} Z_{(3,2)}\right), \\
& Z_{(5,3)}=-\frac{1}{{ }_{5} P_{4}} .
\end{aligned}
$$


From the above equations, we have the generalized term.

$$
\sum_{n=1}^{\infty} \frac{(-1)^{n}}{(2 n-1)^{m}}=\pi^{m}\left\{Z_{(m, 1)} \frac{1}{2^{2}}+Z_{(m, 2)} \frac{1}{2^{4}}+Z_{(m, 3)} \frac{1}{2^{6}}+\cdots+Z_{\left(m, \frac{m+1}{2}\right)} \frac{1}{2^{m+1}}\right\},
$$

where recursively we define (for an odd positive integer $m$ )

$$
\begin{aligned}
& Z_{(m, 1)}=\sum_{k=0}^{\frac{m-3}{2}} \frac{(-1)^{\frac{m-3}{2}+k}}{(m-2 k) !} Z_{(2 k+1,1)}, \\
& Z_{(m, 2)}=\sum_{k=0}^{\frac{m-5}{2}} \frac{(-1)^{\frac{m-5}{2}+k}}{(m-2(k+1)) !} Z_{(2(k+1)+1,2)}, \\
& Z_{(m, 3)}=\sum_{k=0}^{\frac{m-3}{2}} \frac{(-1)^{\frac{m-3}{2}+k}}{(m-2 k) !} Z_{(2(k+2)+1,3)}, \\
& Z_{\left(m, \frac{m-1}{2}\right)}=\frac{1}{3 !} Z_{\left(m-3, \frac{m-1}{2}\right)} \\
& Z_{\left(m, \frac{m+1}{2}\right)}=(-1)^{\frac{m+1}{2}} \frac{1}{m !} .
\end{aligned}
$$

Thus, we have Theorem 3.2.

Theorem 3.3 Let $m$ be a positive odd integer. Then one has

$$
\sum_{n=1}^{\infty} \frac{(-1)^{n}}{(2 n-1)^{m}}=\pi^{m} \sum_{j=1}^{\frac{m-1}{2}}\left(\frac{1}{2^{2 j}} \sum_{k=0}^{\frac{m-(2 j+1)}{2}} \frac{(-1)^{\frac{m-(2 j+1)}{2}+k}}{(m-2(k+j-1)) !} Z_{(2(k+j-1)+1, j)}+Z_{\left(m, \frac{m+1}{2}\right)} \frac{1}{2^{m+1}}\right)
$$

where $Z_{\left(m, \frac{m+1}{2}\right)}=(-1)^{\frac{m+1}{2}} \frac{1}{m !}$.

Example 3.4 By using Theorem 3.3, in case of $m=5$ we have

$$
\begin{aligned}
\sum_{n=1}^{\infty} \frac{(-1)^{n}}{(2 n-1)^{5}} & =\pi^{5} \sum_{j=1}^{\frac{5-1}{2}}\left(\frac{1}{2^{2 j}} \sum_{k=0}^{\frac{5-(2 j+1)}{2}} \frac{(-1)^{\frac{5-(2 j+1)}{2}+k}}{(5-2(k+j-1)) !} Z_{(2(k+j-1)+1, j)}+Z_{\left(5, \frac{5+1}{2}\right)} \frac{1}{2^{5+1}}\right) \\
& =\pi^{5} \sum_{j=1}^{2}\left(\frac{1}{2^{2 j}} \sum_{k=0}^{2-j} \frac{(-1)^{2-j+k}}{(5-2(k+j-1)) !} Z_{(2(k+j-1)+1, j)}+Z_{(5,3)} \frac{1}{2^{6}}\right) \\
& =\pi^{5}\left\{\left(\sum_{k=0}^{1} \frac{(-1)^{k+1}}{(5-2 k) !} Z_{(2(k+1), 1)}\right) \frac{1}{2^{2}}+\left(\frac{1}{3 !} Z_{(3,2)}\right) \frac{1}{2^{4}}+Z_{(5,3)} \frac{1}{2^{6}}\right\} \\
& =\pi^{5}\left\{\left(\frac{-1}{5 !} Z_{(1,1)}+\frac{1}{3 !} Z_{(3,1)}\right) \frac{1}{2^{2}}+\left(\frac{1}{3 !} Z_{(3,2)}\right) \frac{1}{2^{4}}+Z_{(5,3)} \frac{1}{2^{6}}\right\},
\end{aligned}
$$

where $Z_{(5,3)}=(-1)^{3} \frac{1}{5 !}$. 


\section{Competing interests}

The authors declare that they have no competing interests.

\section{Authors' contributions}

Both authors contributed equally to the manuscript and read and approved the final manuscript.

\section{Acknowledgements}

The authors express their gratitude to the referee for his/her valuable comments. We also thank T. Kim and A. Sankaranarayanan for their great support and encouragement. They kindly offered invaluable lecture and advice about the paper. This work was supported by NRF (National Research Foundation of Korea) Grant funded by the Korean Government (NRF-2013-Fostering Core Leaders of the Future Basic Science Program).

\section{Received: 21 April 2013 Accepted: 3 June 2013 Published: 26 June 2013}

\section{References}

1. Kim, T, Choi, J, Kim, YH: A note on the values of Euler zeta functions at the positive integers. Adv. Stud. Contemp. Math. 22(1), 27-34 (2012)

2. Kim, T: Note on the Euler numbers and polynomials. Adv. Stud. Contemp. Math. 17, 131-136 (2008)

3. Kim, T: Euler numbers and polynomials associated with zeta functions. Abstr. Appl. Anal. 2008, Article ID 581582 (2008)

4. Kim, T: Note on the Euler q-zeta functions. J. Number Theory 129(7), 1798-1804 (2009)

5. Kim, T: Identities involving Frobenius-Euler polynomials arising from non-linear differential equations. J. Number Theory 132(12), 2854-2865 (2012)

6. Kim, T: Some identities on the $q$-Euler polynomials of higher order and $q$-Stirling numbers by the fermionic $p$-adic integral on $\mathbb{Z}_{p}$. Russ. J. Math. Phys. 16(4), 484-491 (2009)

7. Kim, T: Barnes-type multiple q-zeta functions and $q$-Euler polynomials. J. Phys. A 43(25), 255201 (2010)

8. Rim, S-H, Kim, T: Explicit $p$-adic expansion for alternating sums of powers. Adv. Stud. Contemp. Math. 14(2), 241-250 (2007)

9. Sankaranarayanan, A: An identity involving Riemann zeta function. Indian J. Pure Appl. Math. 18(9), 794-800 (1987)

10. Ramachandra, K, Sankaranarayanan, A: A remark on $\zeta(2 n)$. Indian J. Pure Appl. Math. 18(10), 891-895 (1987)

11. Kim, M-S, Hu, Su: On p-adic Hurwitz-type Euler zeta functions. J. Number Theory 132, 2977-3015 (2012)

12. Kim, M-S: On the behavior of $p$-adic Euler L-functions. arXiv:1010.198

13. Ayoub, R: Euler and zeta function. Am. Math. Mon. 81, 1067-1086 (1974)

14. Cohen, H, Friedman, E: Raabe's formula for $p$-adic gamma and zeta function. Ann. Inst. Fourier (Grenoble) 58, 363-376 (2008)

15. Koblitz, N: p-Adic Numbers, p-Adic Analysis and Zeta-Functions, 2nd edn. Springer, New York (1984)

16. Tangedal, BA, Young, PT: On $p$-adic multiple zeta function and log gamma functions. J. Number Theory 131, 1240-1257 (2011)

17. Ryoo, CS: On the generalized Barnes type multiple $q$-Euler polynomials twisted by ramified roots of unity. Proc. Jangjeon Math. Soc. 13, 255-263 (2010)

18. Ryoo, CS: A note on the weighted $q$-Euler numbers and polynomials. Adv. Stud. Contemp. Math. 21(1), 47-54 (2011)

19. Walker, JS: Fourier series. In: Encyclopedia of Physical Science and Technology. Academic Press, San Diego (2001)

20. Grosswald, E: Comments on some formulae of Ramnujan. Acta Arith. 21, 25-34 (1972)

21. Grosswald, E: Die Werte der Riemannschen zeta function an ungeraden argumenstellen. Nachr. Akad. Wiss. Gött., 2 1970, 9-13 (1970)

22. Hardy, GH: A formula of Ramanujan. J. Lond. Math. Soc. 3, 238-240 (1928)

23. Zhang, NY: Values of Riemannjan formula and Riemann zeta function at positive odd numbers. Shuxue Jinzhan (Adv. Math. (China)) 12(1), 61-71 (1983)

24. Srivastava, HM: Some rapidly converging series for $\zeta(2 n+1)$. Proc. Am. Math. Soc. 127, 385-396 (1999)

25. Srivastava, HM: Further series representations for $\zeta(2 n+1)$. Appl. Math. Comput. 97, 1-15 (1998)

26. Bender, CM, Orszang, SA: Advanced Mathematical Methods for Scientists and Engineers. McGraw-Hill, New York (1978)

27. Milgram, MS: Integral and series representations of Riemann's zeta function, Dirichelet's eta function and a Medley of related results. arXiv:1208.3429v1 [math.CV]

28. Dwilewicz, RJ, Minac, J: Values of the Riemann zeta function at integers. MATerials MATematics 2009, 6 (2009)

doi:10.1186/1687-1847-2013-182

Cite this article as: Kang and Ryoo: A research on the recursive value and general terms of the analogue Euler zeta function on positive integers. Advances in Difference Equations 2013 2013:182. 